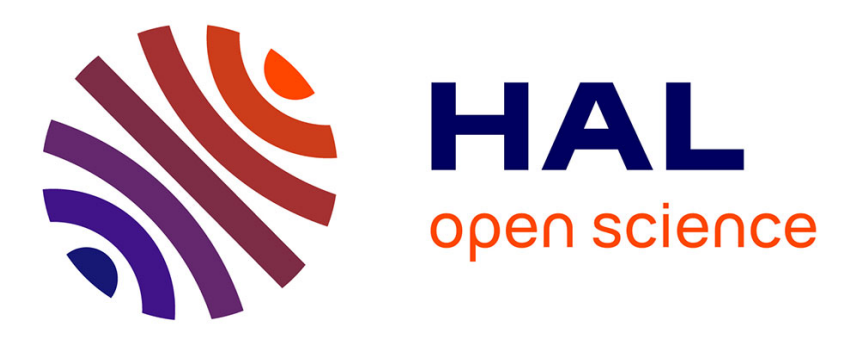

\title{
Ranking orientation responses of path operators: Motivations, choices and algorithmics
}

Odyssée Merveille, Hugues Talbot, Laurent Najman, Nicolas Passat

\section{To cite this version:}

Odyssée Merveille, Hugues Talbot, Laurent Najman, Nicolas Passat. Ranking orientation responses of path operators: Motivations, choices and algorithmics. International Symposium on Mathematical Morphology (ISMM), 2015, Reykjavik, Iceland. pp.633-644, 10.1007/978-3-319-18720-4_53 . hal01168732

\section{HAL Id: hal-01168732 \\ https://hal.science/hal-01168732}

Submitted on 26 Jun 2015

HAL is a multi-disciplinary open access archive for the deposit and dissemination of scientific research documents, whether they are published or not. The documents may come from teaching and research institutions in France or abroad, or from public or private research centers.
L'archive ouverte pluridisciplinaire HAL, est destinée au dépôt et à la diffusion de documents scientifiques de niveau recherche, publiés ou non, émanant des établissements d'enseignement et de recherche français ou étrangers, des laboratoires publics ou privés. 


\title{
Ranking Orientation Responses of Path Operators: Motivations, Choices and Algorithmics ${ }^{\star}$
}

\author{
Odyssée Merveille ${ }^{1,2}$, Hugues Talbot ${ }^{1}$, Laurent Najman $^{1}$, and Nicolas Passat ${ }^{2}$ \\ ${ }^{1}$ Université Paris-Est, ESIEE-Paris, LIGM, CNRS, France \\ ${ }^{2}$ Université de Reims Champagne-Ardenne, CReSTIC, France
}

\begin{abstract}
A new morphological operator, namely RORPO (Ranking Orientation Responses of Path Operators), was recently introduced as a semi-global, morphological alternative to the local, Hessian-based operators for thin structure filtering in $3 \mathrm{D}$ images. In this context, a previous study has already provided experimental proof of its relevance by comparison to such differential operators. In this article, we present a methodological study of RORPO, which completes the presentation of this new morphological filter. In particular, we expose the motivations of RORPO with respect to previous morphological strategies; we present algorithmic developments of this filter and the underlying robust path operator; and we discuss computational issues related to parametricity and time efficiency. We conclude this study by a discussion on the methodological and applicative potentiality of RORPO in various fields of image processing and analysis.
\end{abstract}

Keywords: Antiextensive filtering, robust path openings, thin structure detection, 3D grey-level imaging, vesselness.

\section{Introduction}

Thin structures in $n \mathrm{D}$ images are characterized by a lower size in (at least) one of their $n$ dimensions, compared to the others. Among them, linear structures, i.e.the patterns that present a low size in $n-1$ dimensions, are the most difficult to handle. The difficulty of coping with such patterns increases with the dimension of the surrounding image, as the ratio of the structures volume versus the image volume decreases - resulting in a high sensitivity to noise - while the degrees of freedom of their geometry increases. Dealing with these issues of sparcity and geometry complexity is still tractable in 2D but becomes difficult in 3D.

This has motivated the development of various filtering and segmentation methods for thin 1D structures in the fields of imaging that deal with 3D data, e.g., medical imaging or material sciences [1, Chapters 1,2$]$. In this context, we recently introduced a new filter called RORPO (Ranking Orientation Responses of Path Operators) [2], as a semi-global, discrete, graph-based alternative to the local, continuous, second-order derivative Hessian filter proposed by Frangi et al. [3], and its numerous variants.

\footnotetext{
* This research was funded by the French Agence Nationale de la Recherche (Grant Agreements ANR-10-BLAN-0205 and ANR-12-MONU-0010) and through Programme d'Investissements d'Avenir (LabEx Bézout, ANR-10-LABX-58).
} 
In [2], RORPO was described mainly from a behavioural point of view, motivated against continuous and local approaches, and experimentally validated in the field of 3D medical imaging, where Hessian-based filters are considered the gold standard.

In the present article we now complete our description of RORPO by presenting this operator in the context of graph-based and morphological filters (Section 2), in order to motivate its definition and justify its new contributions with respect to the morphological state of the art (Section 3). Then, we describe algorithmic key-points, hard and soft parametricity and the computational cost of RORPO (Section 4). An illustration of RORPO to materials image denoising and medical image segmentation is proposed (Section 5), and a short discussion of its further uses for segmentation guidance (Section 6) conclude this article, which can be viewed as a companion to [2].

\section{Morphological filtering of 1D structures in grey-level images}

In grey-level images, thin 1D structures can present a complex geometry (e.g., curvature, tortuosity) and a complex topology (e.g., branches, cycles). However, we may assume that they are composed of connected, locally straight segments of locally extremal intensity. These hypotheses induced three main families of approaches.

\subsection{Local (SE-based) approaches}

Following the local straightness and extremal intensity hypotheses, a basic idea is to develop filters from small kernels, fitting these specific properties. In the framework of mathematical morphology, structuring elements (SEs) play the role of such kernels.

Two dual approaches have been considered. The first models a $1 \mathrm{D}$ segment by a small straight SE [4,5], to carry out opening or closing operations by line segments of arbitrary orientation [6,7]. The second models the background in the orthogonal hyperplane of the segment, to carry out grey-level hit-or-miss transforms [8,9].

Even if the shape of a SE is unyielding, some degrees of freedom remain, for instance by varying its orientation. To represent the morphological analogue of a filter bank, several strategies have been experimented with: rotating structuring elements [10], knowledge-based parameterization [8], or content-based spatially-variant mathematical morphology [11]. Blurred operators [9] have been proposed to increase the robustness of the filtering.

Nevertheless, the inflexible geometry of such SEs remains a limitation to the accuracy of these approaches, that progressively led to consider more adaptive SEs [12].

\subsection{Global (connectivity-based) approaches}

Following the global connectedness and locally extremal intensity hypotheses, an alternative approach is to consider connected filters. In the framework of mathematical morphology, the notion of component-tree [13] has been specifically investigated.

In this context, attribute-based methods have been developed for antiextensive filtering. The attributes are mostly scalar $[14,15]$, allowing threshold-based interaction. Recent effort have been directed towards the development of geodesic attributes [16], 
particularly designed for thin structures. Vectorial attributes are less frequently used [17], due to a more complex handling, often requiring a learning step.

The main drawback of connected filters is their unability to split connected components within an image, that may result in erroneous / undesired connections between 1D structures and artifacts, or between 1D branching segments. Various attempts at minimizing these drawbacks have been proposed, either via tilling approaches [17], or with undirected variants of component-trees [18].

It is worth mentionning that hybrid SE / connected strategies have been pursued, e.g., in [19] for reconnection purposes. Links between connectivity-based and pathbased approaches (described below) were also investigated in [20].

\subsection{Semi-global (path-based) approaches}

Following the global and local concepts of connectedness, straightness and extremal intensity hypotheses, approaches based on optimal paths lie at the convergence of the above two families. Global optimal paths consist of finding a series of successive vertices in a graph that minimizes a cost between two points, following standard strategies (e.g., Dijkstra algorithm; see [21] for a recent formal discussion).

In [22], a notion of local optimal path was pioneered. The purpose was to restrict path search to a given distance, and in a given cone of orientations, in order to find the best paths starting from a given point. This paradigm led to the development of a notion of Path Operators (PO) [23], which is an SE-based approach, where the considered SEs are a family of paths i.e., thin elongated connected sets, instead of a fixed shape, thus enabling a higher flexibility in geometry and size, while preserving a 1D semantics.

Algorithmic efforts were conducted to render such an approach dimension-independent [24] and robust to noise $[25,26]$, leading to a notion of Robust Path Operator (RPO). Sparse paradigms [27] were also proposed to avoid redundant computation.

The use of PO and RPO, an a few other path-based paradigms (e.g., polygonal path image [28]), have been proposed in medical imaging and remote sensing [29,30,31], but for $2 \mathrm{D}$ applications only. In the sequel, we recall the basics of $\mathrm{PO}$, and we discuss the challenges related to the 2D-3D transition, which constitute the genesis of RORPO.

\section{Path operators and the 2D-3D transition problem}

\subsection{Path operators: basic notions}

Path operators can be path openings or closings. Without loss of generality, we will discuss the opening case when convenient to avoid unnecessary repetitions.

Binary path operators A binary image $X$ is simply defined as a finite set of points. In the case of path operators, we are interested in the neighbourhood relations between the points of $X$. More precisely, we are interested in the notion of paths, defined as sets of connected points (i.e., successive neighbours). Given an adjacency (non necessarily symmetric) relation $\rightarrow$ on $X$, we can define the set $\Pi_{L}(X)$ of all the paths $\pi$ of the graph 
$(X, \rightarrow)$ of length equal to $L$. The binary path opening $\alpha_{L}(X) \subseteq X$ is defined as the union of all these paths:

$$
\alpha_{L}(X)=\bigcup\left\{\sigma(\pi) \mid \pi \in \Pi_{L}(X)\right\}
$$

where $\sigma(\pi)=\left\{x_{1}, x_{2}, \ldots, x_{L}\right\}$ is the set of points of $X$ forming the path $\pi$.

A binary path opening preserves each point of $X$ belonging to at least one path. From the above definitions, it is plain that $\alpha_{L}$ is increasing, antiextensive, and idempotent; from an algebraic point of view, it is a morphological opening. The $\alpha_{L}$ operator can also be viewed as an SE-based opening. Indeed, following a spatially-variant paradigm, it is an opening with respect to the family of all the SEs that can be defined from the graph $(X, \rightarrow)$. Practically, an elementary graph pattern periodically reproduced over a whole regular set $\Omega \supseteq X$ is used, resulting in a set of SEs that is invariant by translation. For instance, if we set $\Omega \subset \mathbb{Z}^{2}$, and $\rightarrow$ as an arc between $(x, y)$ and $(x, y+1)$, then $\alpha_{L}$ defines the standard opening by a linear vertical SE of $L$ points.

Grey-level path operators A grey-level image $I$ associates a value $I(x) \in \mathbb{R}$ to each point $x$ of a finite set $\Omega$. Practically, such an image is then a function $I: \Omega \rightarrow \mathbb{R}$. The notion of path opening can be easily extended from binary to grey-level images, following a flat morphology paradigm. By applying the binary path opening $\alpha_{L}$ on the binary level sets $I_{\lambda}=\{x \in \Omega \mid I(x) \geq \lambda\}$ of the image $I$, obtained by thresholding at value $\lambda$, we define the grey-level path opening operator $A_{L}$ :

$$
A_{L}(I): x \mapsto \bigvee\left\{\lambda \mid x \in \alpha_{L}\left(I_{\lambda}\right)\right\}
$$

\subsection{The 2D-3D transition problem}

To filter thin structures, PO are parameterized by a given adjacency $\rightarrow$ defined on $\Omega$. As stated above, this adjacency is generally defined from an elementary pattern, reproduced periodically over $\Omega$, leading to a regular graph structure. This pattern allows us to tune the general orientation, but also the tortuosity of the authorised paths by defining locally the angular area where each new point of the path can be defined with respect to its predecessor. For instance, the pattern $(x, y) \rightarrow(x, y+1)$ in $\mathbb{Z}^{2}$, previously evoked, only authorises vertical paths without any tortuosity; by contrast, the pattern $(x, y) \rightarrow\{(x-$ $1, y+1),(x, y+1),(x+1, y+1)\}$, authorises vertical paths within an angular cone of $[\pi / 4,3 \pi / 4]$, around the vertical direction, and a maximal tortuosity of $\pi / 2$ between three successive points.

Filtering approaches based on PO have been recently and successfully applied, e.g., in materials science, remote sensing or medical imaging. These applications $[29,24,30,31]$ have been mostly limited to $2 \mathrm{D}$ images.

Indeed, in $\mathbb{Z}^{2}$, PO preserves the zones of the image in which the paths of authorised orientation, tortuosity and length, can lie; this results in preserving 1D linear structures, plus large $2 \mathrm{D}$ flat zones, the second being easily discriminated from the first by standard opening. In $\mathbb{Z}^{3}$, two kinds of thin structures coexist: $1 \mathrm{D}$ line and 2D plane structures; both are easily discriminated from 3D flat zones. However, PO cannot directly distinguish 1D lines from 2D planes. Indeed, a path lying into a 1D line also lies into a 2D plane in the same orientation. 

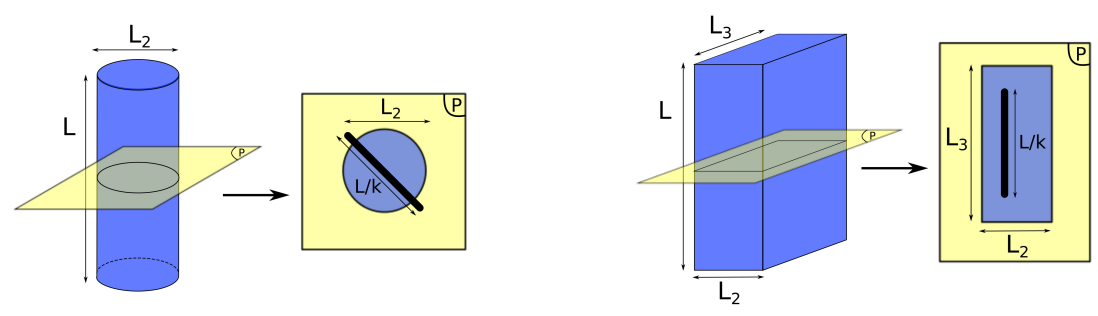

Fig. 1. A segment SE (in black) of length $L / k$ cannot fit a $1 \mathrm{D}$ object (left) but can fit a $2 \mathrm{D}$ one (right), in the plane $P$ orthogonal to the orientation of their largest dimension (see text).

Based on these facts, we now describe the process that led us to define RORPO as the natural way to use PO for 1D thin structure filtering from 3D images.

\subsection{A first approach: Top-Hat path-based filtering}

The main limitation of PO is its unability to discriminate 1D lines from 2D planes. A simple idea consists of applying a post-processing operator for performing such a discrimination. Let us consider an object $X$ of $\mathbb{Z}^{3}$, and let us note $L, L_{2}$ and $L_{3}$ its size in its three principal directions, with $L \geq L_{2} \geq L_{3}$. A 1D line structure will satisfy $L \gg L_{2} \simeq L_{3} ;$ a 2 D plane structure $L \simeq L_{2} \gg L_{3}$, and a 3D structure $L \simeq L_{2} \simeq L_{3}$.

Only 1D objects exhibit a significant difference between $L$ and $L_{2}$. In other words, there exists a value $k>0$ such that $L>k L_{2}$. Let $d$ be the orientation of the 1D structure; a radial opening [7] with a segment SE of size $\frac{L}{k}$ in the plane $P$ orthogonal to $d$, will preserve any structure in $P$ that is longer than $\frac{L}{k}$ (Figure 1). Based on these considerations, applying a top-hat transformation involving such a radial opening on $\alpha_{L}(X)$ should be sufficient to remove the $2 \mathrm{D}$ planes while preserving the $1 \mathrm{D}$ structures.

This approach, although simple, presents several drawbacks. The first is quantitative: an object is considered 1D if it is at least $k$ times longer than larger, but this parametric value $k$ is not necessarily easy to tune. The second is geometric: the directions of the path operators are defined by angular cones. Nevertheless, the (radial) opening is not as flexible as PO: it is performed only in one plane $P$, namely the one orthogonal to the central axis of the cone. This may induce approximate or erroneous results. The third drawback is computational: calculating the radial openings is significantly costlier than the PO itself.

\subsection{RORPO filtering}

We now seek a strategy that allows us not only to detect thin structures, but also to discriminate their 1D nature in a robust, non-parametered way, and without requiring costly post-processing. A key-idea that underlies the RORPO filter is to use PO both for detecting thin structures and characterizing them as $1 D$.

We now consider a grey-level image $I: \Omega \rightarrow \mathbb{R}$ and a point $x$ of $\Omega$. The grey-level path operator $A_{L}^{d}(I)$ for the direction $d$ should associate a value $A_{L}^{d}(I)(x) \simeq I(x)($ resp. a 


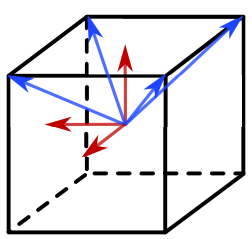

(a)

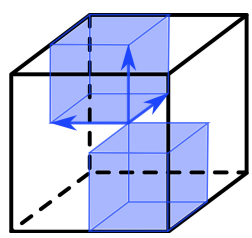

(e)

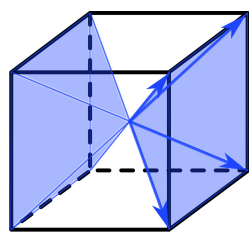

(b)

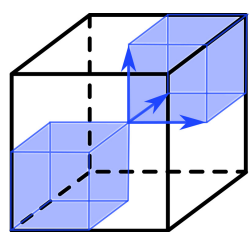

(f)

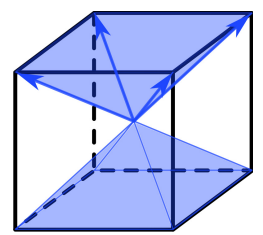

(c)

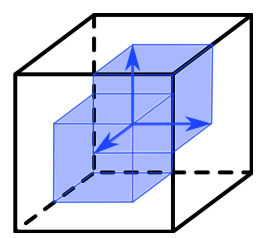

(g)

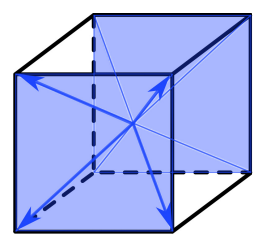

(d)

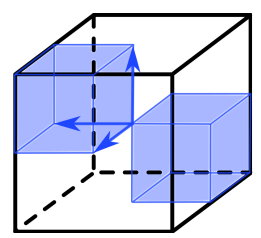

(h)

Fig. 2. (a) The 7 orientation vectors of $\mathcal{D}$ (principal orientations in red, principal diagonals in blue). The cones of orientations associated to the red vectors $(b-d)$ and to the blue vectors $(e-h)$.

value $\left.A_{L}^{d}(I)(x) \ll I(x)\right)$ if the direction $d$ lies inside (resp. outside) of a thin structure. The rationale of RORPO filter, noted $\gamma_{L}$, consists of determining the status of $x$ by only analysing the set of PO responses $\left\{A_{L}^{d}(I)(x)\right\}_{d \in \mathcal{D}}$ for the set of orientations $\mathcal{D}$.

In theory, the analysis of these responses requires on the one hand a set $\mathcal{D}$ providing a dense, isotropic sampling of the 3D orientations; and on the other hand a qualitative analysis of the $A_{L}^{d}(I)(x)$ responses. In practice, when working on $\mathbb{Z}^{3}$, it is not tractable to define a large number of orientations. In the proposed version of RORPO [2], it was chosen to define 7 cones of orientations, corresponding to the 3 principal directions of $\mathbb{Z}^{3}$, plus the 4 principal diagonals (Figure 2). This choice is discussed in Section 4.2.

Our working hypothesis is that a quantitative analysis of $\left\{A_{L}^{d}(I)(x)\right\}_{d \in \mathcal{D}}$ should then be sufficient to decide wether $x$ belongs to a thin 1D structure in $I$. Indeed, a 2D structure, being elongated in one more direction than a 1D structure, should be detected in more orientations $d \in \mathcal{D}$ than a 1D one. In order to experimentally confirm this intuition, we computed the number of high responses, $\left\{A_{L}^{d}(I)(x)\right\}_{d \in \mathcal{D}}$, for 100 synthetic binary straight tubes, planes and curved planes, respectively. The dimensions of the synthetic binary structures was set according to the fixed $L$ chosen as the point of this experiment is to determine the influence of the direction. Results (Figure 3) tend to validate this conjecture. Indeed, 1D structures are detected in at most 3 out of the 7 orientations of $\mathcal{D}$ whereas 2D structures are detected in at least 4 .

As a consequence, the discrimination between $1 \mathrm{D}$ and 2D objects in practice consist of sorting the $7\left\{A_{L}^{d}(I)(x)\right\}_{d \in \mathcal{D}}$, and defining the RORPO output as the difference between the highest (1st) and median (4th) values. The formula of RORPO is then simply defined as

$$
\gamma_{L}(I)(x)=\gamma_{L}^{1}(I)(x)-\gamma_{L}^{4}(I)(x)
$$

where $\gamma_{L}^{i}(I)(x)(i \in[1,7])$ is the $i$ th greatest value within $\left\{A_{L}^{d}(I)(x)\right\}_{d \in \mathcal{D}}$. 

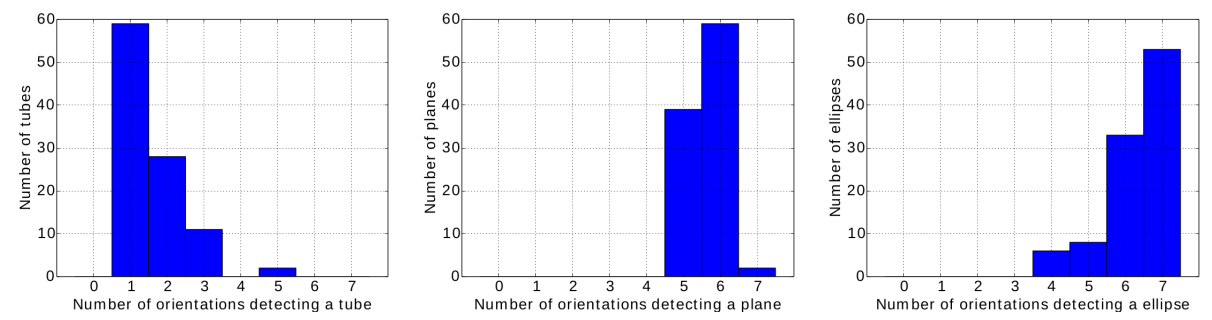

Fig. 3. Ratio of path opening high responses, computed from 100 binary synthetic examples: (left) a tube, (center) a plane, and (right) a curved plane, within the set $\mathcal{D}$ of 7 orientations illustrated in Figure 2.

\section{Algorithmics, parameters and computational cost}

\subsection{Robust path-opening}

The core of RORPO is the computation of several path openings. RORPO then inherits the main weakness of PO, that is its non-robustness to disconnections induced by noise, a fortiori for high values of $L$. This fact motivated the development of algorithms for robust path-opening (RPO). Pioneering works were conducted in $[23,25]$, and were finalised by the proposal of a RPO method by Cokelaer et al. in [26]. The principle is to tolerate $K$ disconnected ("noisy") points between two successive points of the path.

From an applicative point of view, Cokelaer's RPO provides good results. Nevertheless, noisy points are not taken into account for determining the overall path length. The detected paths then have lengths between $L$ (noise-free) and $L+K(L-1)$ (the most noisy). In this context, the parameter $L$ becomes meaningless. In addition, the algorithmic layer devoted to handle the robustness is time and memory consuming.

Two alternatives of RPO were investigated. The first consists of explicitly modeling the jump between two non-noisy points via the adjacency relation, by considering the $K$-th power of $\rightarrow$, namely $\rightarrow^{K}$, instead of $\rightarrow$. This preserves $L$, but leads to complex and still time-consuming adaptations of PO processing; The second approach relies on a mask-based second-connectivity strategy [32], in order to "reconnect" the noisy parts of the graphs. Such approach was already proposed for 3D vessel segmentation in the framework of spatially-variant mathematical morphology [19]. The idea is here more basic and non-costly - but still effective - as a dilation by a cubical SE of size $K+1$ is performed on the initial image $I$. In order to preserve the anti-extensivity of PO, an infimum operator is finally applied:

$$
A_{L}^{K}(I)=\bigwedge\left\{I, A_{L}\left(\delta_{K+1}(I)\right)\right\}
$$

This approach, although not equivalent to [26] from a theoretical point of view, is quite similar in terms of results. As a benefit, it preserves the correct path length. It presents a computational cost that is equivalent to that of $\mathrm{PO}$ (only additional linear time operations are required), and is much lower than [26]. 


\subsection{Parameters}

RORPO depends on a small number of parameters, which constitutes one of its strengths. These few hard and soft parameters are listed and discussed hereafter.

Orientation sampling Working in a discrete space implies to choose a sampling policy to determine the number and the shape of the cones for computing the (R)POs. Considering the structure of $\mathbb{Z}^{3}$, isotropy requirements, and the algorithmic constraints of PO, three main families of sampling policies may be considered: along the 3 principal orientations; along the $3+4$ principal orientations and principal diagonals; and along the $3+4+6$ principal orientations and principal / secondary diagonals. It is plain that 3 orientations are not enough to accurately capture 1D structures. As discussed in Section 3.4 and [2], 7 orientations were experimentally proved to be sufficient to obtain satisfactory results. Experiments were also carried out to assess the relevance of considering 13 orientations. Three main reasons finally led us to reject this possibility. First, increasing the number of orientations complexifies a quantitative analysis necessary to define a simple formula similar to Equation (3). Second, the multiplication of angular cones also multiplies the handling of "limit cases" (see below). Third, the number of PO computations is nearly doubled, for a limited benefit.

Angular cones The basic patterns for each of the 7 orientations have to fully cover the direct neighbourhood of any point $(x, y, z)$ of $\mathbb{Z}^{3}$, namely, the 26 points forming a $3 \times 3 \times 3$ cube around $(x, y, z)$. Two policies may be considered: choosing patterns that induce either a partition or a cover of these 26 points. The main advantage of a partition is to avoid some overlapping effects between the angular cones, that may induce undesired side effects. Unfortunately, such a policy is not acceptable as it omits some paths whose global orientation lies in between the bounds of two successive angular cones. (For instance, in $\mathbb{Z}^{2}$, two successive patterns $(x, y) \rightarrow\{(x+1, y),(x+1, y+1)\}$ and $(x, y) \rightarrow\{(x, y+1),(x-1, y+1)\}$ generate graphs where some paths of orientation within $] \pi / 4, \pi / 2$ [ will not be considered). It was then chosen to adopt a covering policy. In order to respect isotropy requirements and minimal overlapping, this cover was defined as illustrated in Figure 2(b-h). The counterpart of covering is the existence of limit cases, that correspond to the paths of length $L$ that lie exactly at the frontier separating several cones. In such limit cases, Equation (3) is not valid. To correct this problem, a low cost but specific processing was devised (see [2] for a discussion on this topic).

Robustness parameter In our robust version of PO, the tolerance to disconnections, i.e., the maximal number of noisy points between two successive vertices of the path, is directly linked to the size of the cubical SE used for the dilation of the filtered image. We chose to fix this size to 3 , i.e., $K=2$, in order to allow a path propagation through a maximum of 2 successive noisy pixels. Setting this size to a larger value would increase the number of false propagations and the background noise reduction would be less effective. 

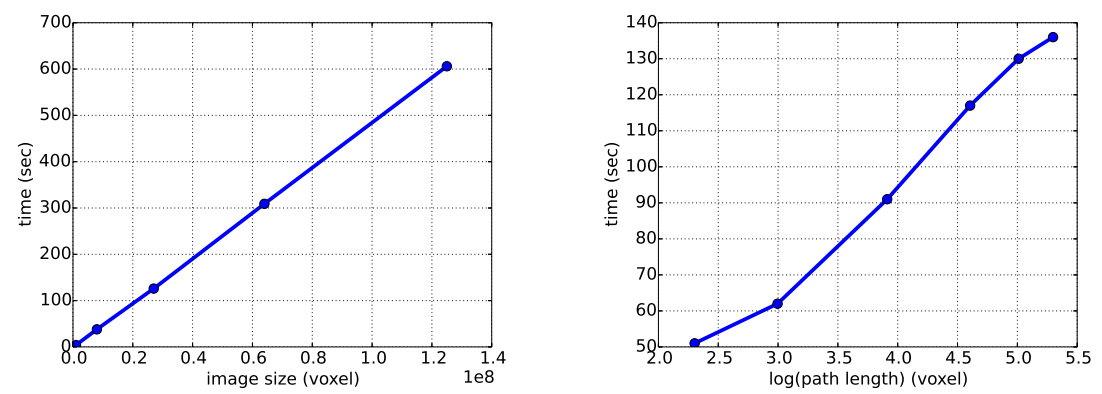

Fig. 4. Computational cost of RORPO, with respect to $|\Omega|$ (left) and $L$ (right, log scale).

Path length The path length $L$ is the only real (i.e., tunable) parameter of RORPO. Since it corresponds to the minimal length of the paths that lie in a given angular cone, it carries both a size and a curvature information. More precisely, when increasing $L$, RORPO will discard both small 1D structures, but also tortuous 1D structures whose part lying in a same angular cone does not exceed a length $L$. The choice of $L$ should then derive from a trade-off between these two criteria.

\subsection{Space and computational cost}

In terms of memory usage, RORPO requires storing 7 images $A_{L}^{d}(I)$ obtained from the RPOs computed from $I$. Since only the maximal and median of these 7 values are finally required at each point, it is possible to optimize the process, to only store the first 4 images $\gamma_{L}^{i}(I)$ useful for computing $\gamma_{L}(I)$. The overall space cost is then $4 .|\Omega|$, plus the cost of the chosen (R)PO method.

In terms of computational cost, the first step of the method consists of implementing Equation (4). The dilation, and infimum operations have linear cost $O(|\Omega|)$ with respect to the size of the image $I$. The time cost of this step is then dominated by that of the PO method, noted $T_{P O}$. The second step, that implements Equation (3), requires to access the sorted values of the 7 filtered images. This sorting has a linear cost $O(|\Omega|)$, and so has the step. The handling of limit cases involves a final step that mainly relies on greyscale reconstructions [33] on filtered images $\gamma_{L}(I)$, and also leads to a linear cost $O(|\Omega|)$. Finally, the total time cost of the method ${ }^{1}$ is of the same order as 7.T $T_{P O}$. Between the algorithms proposed in the literature $[25,24]$, we considered the latter, designed by Hendriks, that provides better genericity.

The experimental computation times gathered in Figure 4 assess the behaviour of RORPO with respect to the size $|\Omega|$ of the image and the length parameter $L$. These experiments were performed on synthetic vascular networks generated by Vascusynth [34] which allows us to tune the network density and image size independently. These results confirm that the time complexity is linear with respect to $|\Omega|$ (Figure 4(a)) and logarithmic with respect to $L$ (Figure 4(b)).

\footnotetext{
${ }^{1}$ The $\mathrm{C}++$ code of RORPO is available at: http://path-openings.github.io/RORPO.
} 


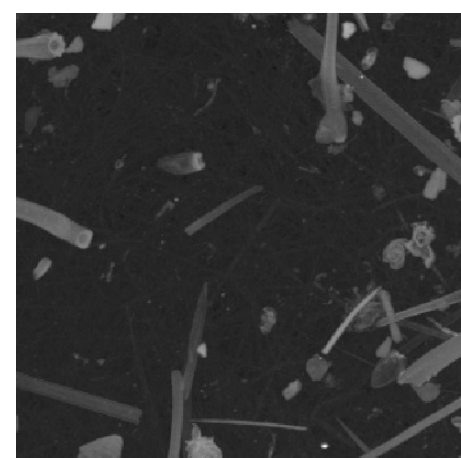

(a)

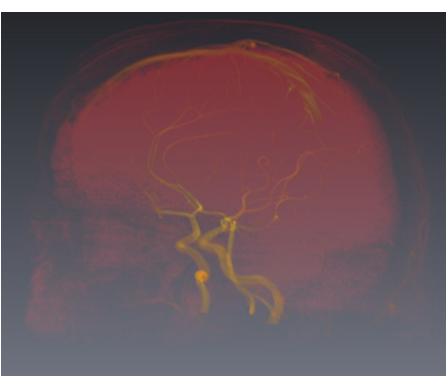

(c)

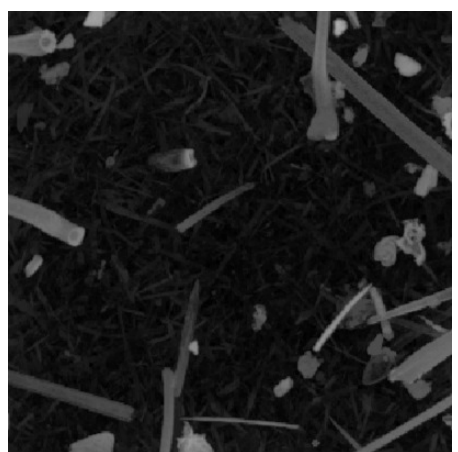

(b)

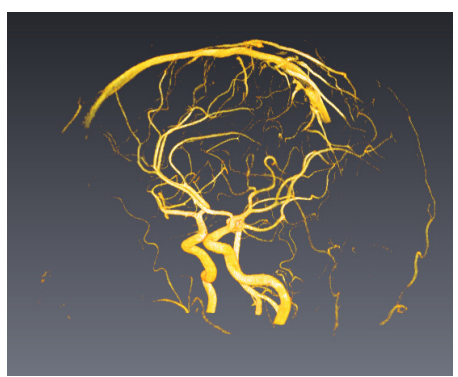

(d)

Fig. 5. A high resolution fibre image before (a) and after (b) RORPO filtering (maximum intensity projection). Brain magnetic resonance angiography (c) and component-tree segmentation with automatic markers selection using RORPO results (d) (volume rendering). (a) Courtesy Sébastien Moulinet, LPS, UMR 8550. (c) Courtesy In-Vivo Imaging Platform, Strasbourg University.

\section{Illustration}

Medical applications, both on coronary arteries and brain vessels, were presented in [2]. We provide here an illustration of filtering by RORPO on a micro-CT fibre volume. Results show that small fibres which were hidden in the background noise in the initial image (Figure 5(a)) appear distinctly in the filtered image (Figure 5(b)).

As a low-level filter, information enhanced by RORPO may be used for the guidance of segmentation frameworks. Figure 5(c-d) presents results of a component-tree segmentation [35] using markers resulting from a threshold of the RORPO response. This automatic marker selection dispenses from manually selecting markers, a tedious task in the case of a complex structure as the brain vascular network.

\section{Conclusion}

This article, together with [2], provides a comprehensive description of RORPO as an efficient 1D structure detection filter. From a filtering point of view, the main perspec- 
tives are related to the handling of branching points (not dealt with here, as RORPO is a pure tube detector), and to the potential extension of RORPO in scale-space paradigms.

From a segmentation point of view, RORPO may be used as a guiding term in various energy-based, statistical or learning approaches for 3D image processing. In particular, research efforts have been initiated in the fields of Markovian and variational segmentation of 3D angiographic images.

From an applicative point of view, some extensions of RORPO may also be considered for explicitly detecting $2 \mathrm{D}$ thin structures. In this context, it may be compared to other recently proposed approaches [36] toward this goal in mathematical morphology.

\section{References}

1. Talbot, H.: Étude des directions en analyse d'image. Habilitation Thesis, Université Paris-Est (2013) (In English).

2. Merveille, O., Talbot, H., Najman, L., Passat, N.: Tubular structure filtering by ranking orientation responses of path operators. In: ECCV, Proc. Volume 8690 of LNCS., Springer (2014) 203-218

3. Frangi, A.F., Niessen, W.J., Vincken, K.L., Viergever, M.A.: Multiscale vessel enhancement filtering. In: MICCAI, Proc. Volume 1496 of LNCS., Springer (1998) 130-137

4. Zana, F., Klein, J.C.: Segmentation of vessel-like patterns using mathematical morphology and curvature evaluation. IEEE Transactions on Image Processing 10(7) (2001) 1010-1019

5. Tankyevych, O., Talbot, H., Dokládal, P.: Curvilinear morpho-Hessian filter. In: ISBI, Proc. (2008) 1011-1014

6. Soille, P., Breen, E., Jones, R.: Recursive implementation of erosions and dilations along discrete lines at arbitrary angles. IEEE Transactions on Pattern Analysis and Machine Intelligence 18(5) (1996) 562-567

7. Soille, P., Talbot, H.: Directional morphological filtering. IEEE Transactions on Pattern Analysis and Machine Intelligence 23(11) (2001) 1313-1329

8. Naegel, B., Passat, N., Ronse, C.: Grey-level hit-or-miss transforms-Part II: Application to angiographic image processing. Pattern Recognition 40(2) (2007) 648-658

9. Bouraoui, B., Ronse, C., Baruthio, J., Passat, N., Germain, P.: 3D segmentation of coronary arteries based on advanced mathematical morphology techniques. Computerized Medical Imaging and Graphics 34(5) (2010) 377-387

10. Thackray, B.D., Nelson, A.C.: Semi-automatic segmentation of vascular network images using a rotating structuring element (ROSE) with mathematical morphology and dual feature thresholding. IEEE Transactions on Medical Imaging 12(3) (1993) 385-392

11. Tankyevych, O., Talbot, H., Dokládal, P., Passat, N.: Spatially variant morpho-Hessian filter: Efficient implementation and application. In: ISMM, Proc. Volume 5720 of LNCS., Springer (2009) 137-148

12. Buckley, M., Talbot, H.: Flexible linear openings and closings. In: ISMM, Proc. Volume 18 of CIV., Springer (2000) 109-118

13. Salembier, P., Oliveras, A., Garrido, L.: Antiextensive connected operators for image and sequence processing. IEEE Transactions on Image Processing 7(4) (1998) 555-570

14. Wilkinson, M.H.F., Westenberg, M.A.: Shape preserving filament enhancement filtering. In: MICCAI, Proc. Volume 2208 of LNCS., Springer (2001) 770-777

15. Xu, Y., Géraud, T., Najman, L.: Two applications of shape-based morphology: Blood vessels segmentation and a generalization of constrained connectivity. In: ISMM, Proc. Volume 7883 of LNCS., Springer (2013) 390-401 
16. Morard, V., Decencière, E., Dokládal, P.: Efficient geodesic attribute thinnings based on the barycentric diameter. Journal of Mathematical Imaging and Vision 46(1) (2013) 128-142

17. Caldairou, B., Naegel, B., Passat, N.: Segmentation of complex images based on componenttrees: Methodological tools. In: ISMM, Proc. Volume 5720 of LNCS., Springer (2009) 171180

18. Perret, B., Cousty, J., Tankyevych, O., Talbot, H., Passat, N.: Directed connected operators: Asymmetric hierarchies for image filtering and segmentation. IEEE Transactions on Pattern Analysis and Machine Intelligence (DOI:10.1109/TPAMI.2014.2366145).

19. Dufour, A., Tankyevych, O., Naegel, B., Talbot, H., Ronse, C., Baruthio, J., Dokládal, P., Passat, N.: Filtering and segmentation of 3D angiographic data: Advances based on mathematical morphology. Medical Image Analysis 17(2) (2013) 147-164

20. Wilkinson, M.H.F.: Hyperconnectivity, attribute-space connectivity and path openings: Theoretical relationships. In: ISMM, Proc. Volume 5720 of LNCS., Springer (2009) 47-58

21. Stawiaski, J.: Optimal path: Theory and models for vessel segmentation. In: ISMM, Proc. Volume 6671 of LNCS., Springer (2011) 417-428

22. Vincent, L.: Minimal path algorithms for the robust detection of linear features in gray images. In: ISMM, Proc. Volume 12 of CIV., Springer (1998) 331-338

23. Heijmans, H.J.A.M., Buckley, M., Talbot, H.: Path openings and closings. Journal of Mathematical Imaging and Vision 22(2-3) (2005) 107-119

24. Luengo Hendriks, C.L.: Constrained and dimensionality-independent path openings. IEEE Transactions on Image Processing 19(6) (2010) 1587-1595

25. Talbot, H., Appleton, B.: Efficient complete and incomplete path openings and closings. Image and Vision Computing 25(4) (2007) 416-425

26. Cokelaer, F., Talbot, H., Chanussot, J.: Efficient robust d-dimensional path operators. IEEE Journal of Selected Topics in Signal Processing 6(7) (2012) 830-839

27. Morard, V., Dokládal, P., Decencière, E.: Parsimonious path openings and closings. IEEE Transactions on Image Processing 23(4) (2014) 1543-1555

28. Bismuth, V., Vaillant, R., Talbot, H., Najman, L.: Curvilinear structure enhancement with the polygonal path image - Application to guide-wire segmentation in X-ray fluoroscopy. In: MICCAI, Proc. Volume 7511 of LNCS., Springer (2012) 9-16

29. Valero, S., Chanussot, J., Benediktsson, J.A., Talbot, H., Waske, B.: Advanced directional mathematical morphology for the detection of the road network in very high resolution remote sensing images. Pattern Recognition Letters 31(10) (2010) 1120-1127

30. Rossant, F., Badellino, M., Chavillon, A., Bloch, I., Paques, M.: A morphological approach for vessel segmentation in eye fundus images, with quantitative evaluation. Journal of Medical Imaging and Health Informatics 1(1) (2011) 42-48

31. Sigurðsson, E.M., Valero, S., Benediktsson, J.A., Chanussot, J., Talbot, H., Stefánsson, E.: Automatic retinal vessel extraction based on directional mathematical morphology and fuzzy classification. Pattern Recognition Letters 47 (2014) 164-171

32. Ouzounis, G.K., Wilkinson, M.H.F.: Mask-based second-generation connectivity and attribute filters. IEEE Transactions on Pattern Analysis and Machine Intelligence 29(6) (2007) 990-1004

33. Vincent, L.: Morphological grayscale reconstruction in image analysis: Applications and efficient algorithms. IEEE Transactions on Image Processing 2(2) (1993) 176-201

34. Hamarneh, G., Jassi, P.: Vascusynth: Simulating vascular trees for generating volumetric image data with ground truth segmentation and tree analysis. Computerized Medical Imaging and Graphics 34(8) (2010) 605-616

35. Passat, N., Naegel, B., Rousseau, F., Koob, M., Dietemann, J.L.: Interactive segmentation based on component-trees. Pattern Recognition 44(10-11) (2011) 2539-2554

36. Urbach, E.R., Pervukhina, M., Bischof, L.: Segmentation of cracks in shale rock. In: ISMM, Proc. Volume 6671 of LNCS., Springer (2011) 451-460 